Revista Arbitrada Interdisciplinaria KOINONIA

Año VI. Vol VI. N³. Edición Especial: Educación II. 2021

Hecho el depósito de Ley: FA2016000010 ISSN: 2542-3088

FUNDACIÓN KOINONIA (F.K). Santa Ana de Coro. Venezuela.

Karla Esthefanía Jaén-Armijos; Sandra Elizabeth Mena-Clerque

http://dx.doi.org/10.35381/r.k.v6i3.1307

\title{
Alfabetización informacional para el desarrollo de competencias digitales en Educación Básica Media
}

Information literacy for the development of digital skills in Middle Basic Education

\author{
Karla Esthefanía Jaén-Armijos \\ karla.jaen.35@est.ucacue.edu.ec \\ Universidad Católica de Cuenca, Azogues \\ Ecuador \\ https://orcid.org/0000-0002-1015-4586 \\ Sandra Elizabeth Mena-Clerque \\ sandramena@ucacue.edu.ec \\ Universidad Católica de Cuenca, Cuenca \\ Ecuador \\ https://orcid.org/0000-0002-9186-2161
}

Recepción: 10 de abril 2021

Revisado: 05 de mayo 2021

Aprobación: 30 de junio 2021

Publicación: 15 de julio 2021 
Revista Arbitrada Interdisciplinaria KOINONIA

Año VI. Vol VI. N³. Edición Especial: Educación II. 2021

Hecho el depósito de Ley: FA2016000010

ISSN: 2542-3088

FUNDACIÓN KOINONIA (F.K). Santa Ana de Coro. Venezuela.

Karla Esthefanía Jaén-Armijos; Sandra Elizabeth Mena-Clerque

\title{
RESUMEN
}

La alfabetización Informacional es fundamental para mejorar la calidad de la educación puesto que, apropiarse de competencias informacionales permitirá que muchos estudiantes alcancen los niveles de pensamiento superior. En ese sentido, el presente artículo tiene como objetivo analizar el nivel de conocimiento sobre Alfabetización Informacional en los estudiantes y docentes de la Escuela de Educación Básica Particular Hispanoamericano, para desarrollar competencias digitales en el uso y gestión de la información. La metodología asumida es de tipo descriptiva no experimental, con un enfoque mixto. Como resultado de la aplicación de encuestas a padres de familia, entrevistas a docentes de quinto, sexto, séptimo Educación General Básica y directivos de la institución se evidencia un deficiente proceso para buscar, analizar, localizar y evaluar la información adecuadamente. Por lo tanto, ante el desconocimiento de competencias informacionales se ha planteado el Modelo KALFIN 4 con un enfoque innovador y adaptable al contexto educativo.

Descriptores: Alfabetización informacional; competencias para la vida; tecnología de la información; educación básica; gestión del conocimiento. (Palabras tomadas del Tesauro UNESCO).

\begin{abstract}
Information literacy is essential to improve the quality of education since, appropriating informational competencies will allow many students to reach higher levels of thinking. In this sense, the present article aims to analyze the level of knowledge about Information Literacy in students and teachers of the Spanish-American Private Basic Education School, to develop digital skills in the use and management of information. The assumed methodology is descriptive, non-experimental, with a mixed approach. As a result of the application of surveys to parents, interviews with fifth, sixth, and seventh grade teachers, General Basic Education and directors of the institution, there is evidence of a deficient process for searching, analyzing, locating and evaluating the information properly. Therefore, given the lack of knowledge of informational competences, the KALFIN 4 Model has been proposed with an innovative approach that is adaptable to the educational context.
\end{abstract}

Descriptors: Information literacy; life skills; information technology; basic education; knowledge management. (Words taken from the UNESCO Thesaurus). 


\section{INTRODUCCIÓN}

En la actualidad, con los vertiginosos avances tecnológicos a nivel mundial resulta imprescindible el manejo y uso de herramientas digitales, pues con ello el internet ha revolucionado los paradigmas de búsqueda, interacción y gestión de la información. En Ecuador, por ejemplo, se evidencia que, en los últimos tres años ha mejorado el índice de disponibilidad de tecnologías y acceso a internet. Todo eso demuestra la necesidad de alfabetizarnos digitalmente para promover mejores oportunidades.

Así pues, resulta indudable que las tecnologías de información y comunicación (TIC) tienen un gran impacto en la actualidad, pues han transformado las relaciones culturales, educativas, políticas, y socioeconómicas de los países. Dentro del ámbito educativo aquellos niños y jóvenes que no puedan acceder a entornos virtuales/ digitales no serán capaces de participar activamente en contextos económicos y culturales de su vida diaria (Gutiérrez et al. 2016).

Dentro del marco legal educativo específicamente en el literal j, artículo 6, de la (Ley Orgánica De Educación Intercultural, 2016), establece como una de las obligaciones del Estado ecuatoriano "Garantizar la alfabetización digital y uso de las TIC en la educación del Ecuador propiciando el vínculo de la enseñanza con actividades productivas y sociales". Sin embargo, dichas competencias digitales son desarrolladas parcialmente en algunas Instituciones educativas del País porque dentro de la malla curricular del Ministerio de Educación no se exige la enseñanza de "computación" como asignatura obligatoria sino como eje transversal en las demás asignaturas.

Es por ese motivo que, los niños alcanzan años superiores de educación sin saber el manejo programas utilitarios como Word, Excel, PowerPoint, el encendido de una computadora, el acceso a fuentes de información confiables y la realización una correcta investigación. Desde ese contexto, y luego de un profundo análisis de aproximadamente un año, la problemática actual es que los niños de la Escuela de Educación Básica Particular Hispanoamericano subnivel media a pesar de recibir la asignatura de computación no han desarrollado competencias digitales en el manejo y uso de la 
información, pues los niños de quinto, sexto y séptimo año de Educación General Básica no buscan información en la web de manera pertinente, no leen y analizan la información para seleccionarla, los niños simplemente abren el navegador y lo primero que encuentran "copian y pegan" para la investigación.

Dicho esto, resulta necesario implementar la enseñanza de Alfabetización Informacional en todos los niveles educativos, esto permitirá a los estudiantes una formación en conocimientos propios de la búsqueda, análisis, gestión y aplicación de la información tanto a nivel personal como académico y un futuro profesional. Para (González-López et al. 2019), enseñar alfabetización informacional en educación básica permite promover la mejora continua y permanente del aprendizaje, diferenciar fuentes de información para seleccionar la más confiable y verídica, y forma personas críticas.

En definitiva, es fundamental que los niños aprendan a buscar, localizar, seleccionar, y analizar la información que se encuentra en internet. Además de las ventajas propuestas por los autores anteriormente, la enseñanza de la alfabetización informacional permite alcanzar niveles de pensamiento superior y el desarrollo de competencias científicas en los estudiantes.

De igual manera, la emergencia sanitaria de la COVID 19 tanto padres de familia como estudiantes vieron en la necesidad de aprender algunas herramientas digitales importantes para acceder a las clases virtuales y poder resolver tareas. Sin embargo, hablar de Alfabetización Informacional es ir más allá, se trata del acceso, comprensión y evaluación de la información para aplicarla de manera eficaz, crítica y creativa.

Por ese motivo el objetivo general de la investigación es analizar el nivel de conocimiento sobre Alfabetización Informacional en los estudiantes y docentes de Educación Básica subnivel Media de la Escuela de Educación Básica Particular Hispanoamericano para promover el desarrollo de competencias digitales. Para ello es necesario, realizar un diagnóstico de la situación real de los actores educativos y proponer un modelo para implementar la Alfabetización Informacional en la Institución educativa de tal manera que sea referencia para todas las escuelas del cantón Santa Rosa. 
Revista Arbitrada Interdisciplinaria KOINONIA

Año VI. Vol VI. N³. Edición Especial: Educación II. 2021

Hecho el depósito de Ley: FA2016000010 ISSN: 2542-3088

FUNDACIÓN KOINONIA (F.K). Santa Ana de Coro. Venezuela.

Karla Esthefanía Jaén-Armijos; Sandra Elizabeth Mena-Clerque

\section{Referencial teórico}

Aunque el tema de la alfabetización digital por sí solo es popular y comúnmente investigado, los artículos dedicados a su definición son limitados (Chetty et al. 2018), por otro lado, Lucas \& Calle (2019), indican que la Alfabetización Informacional como "information literacy" término empleado para referirse al desarrollo de habilidades informativas en las personas" p.70. Es decir, tener la capacidad para saber cuándo, cómo y porqué se necesita la información. No simplemente es ingresar a un navegador, colocar el tema y escoger lo primero que se encuentra, su utilización necesita de las capacidades para leer, analizar y evaluar la información.

Es importante mencionar que, la alfabetización Informacional es una acción educativa orientada a que los sujetos promuevan en sí mismos procesos de pensamiento, análisis, que tengan la posibilidad de cambiar actitudes frente a la información que encuentran y así mejorar la perspectiva de vida (Morales-Camargo, 2020). Dicho en otras palabras, que aprendan a aprender habilidades para su convivir diario. Esto es importante porque al presentarse una situación difícil o una simple curiosidad se va a pensar dos veces si la primera información que se encuentra es verídica o no, eso generará un pensamiento crítico en el individuo.

Por consiguiente, una buena práctica informacional propiciará un aprendizaje significativo en la persona que lo aplica, sea estos niños, adultos, jóvenes, estudiantes, universitarios o profesionales. Porque no se trata de aprender alfabetización al finalizar un ciclo de estudio, este debe darse a lo largo del proceso enseñanza aprendizaje, porque siempre surgirán problemas que demanden una o más soluciones, por ende, muchas fuentes de información.

Quizás, ese sea el inconveniente, muchas fuentes de información provoca que los estudiantes no realicen procesos de análisis para certificar la fiabilidad de fuentes procedentes de la red (Fernandez-Aragones, \& Camon, 2020). Una de las causas de ese problema además del desconocimiento de las técnicas y herramientas para la búsqueda de información es la falta de lectura en los estudiantes, porque para elegir una 
información es necesaria la lectura reflexiva, donde las ideas sean comprendidas, comparadas y evaluadas a fin de elergir o conseguir otra fuente de información.

A los estudiantes les da pereza leer, peor aún si se trata de un artículo o libro digital. Según (Recio-Pineda, 2020), existe un poco interés hacia la lectura, algunos estudiantes lo consideran un castigo o una actividad muy aburrida. Desde ese punto de vista, resulta difícil enseñar alfabetización informacional a los estudiantes porque todo el proceso para seleccionar y criticar la información encontrada exige un riguroso apego hacia la lectura, y eso limita uno de los objetivos principales de la Alfabetización informacional, el aprendizaje significativo.

Dentro de los objetivos de la Alfabetización Informacional se encuentran: desarrollar competencias relacionadas con la optimización de la búsqueda de información, definición adecuada de conceptos y términos, evaluación reflexiva, y un mejor manejo de la información para consultas, tareas y trabajos de investigación. En la actualidad vivimos en una sociedad donde el conocimiento está en constante cambio, es por eso importante aprender sobre Alfabetización Informacional y otras bondades de las Tecnologías de la Información y comunicación para ser individuos creativos y analíticos.

Cabe entonces señalar ¿Qué rol cumple las TIC en los actuales momentos? Considerando que, son tecnologías propias de la gestión y transformación de la información. Para (García-Ávila, 2016), los medios de comunicación y las tecnologías de la información y de la comunicación (TIC) se encuentran en todos lados y brindan posibilidades para el ciudadano común, que hasta hace poco solo podían imaginarse. Por lo tanto, ser fundamental ser conscientes de la realidad y que la mejor forma de afrontar los retos es desarrollar estrategias que permitan formar ciudadanos de bien, que contribuyan al uso eficiente y ético de la información aprovechando entre ellas los beneficios de la Alfabetización informacional.

Ahora bien, una vez determinados los fundamentos teóricos sobre Alfabetización Informacional y sus ventajas es de gran importancia analizar si dentro del Marco Legal Ecuatoriano se garantiza un eficiente manejo de la información, pues son desde las 
políticas y leyes públicas que se puede innovar en el campo educativo. Partiendo desde la (Constitución de la Republica del Ecuador, 2008), en el artículo 16 establece que todas las personas tienen acceso universal a las tecnologías de información y comunicación. Y el artículo 347, literal 8 señala como una de las responsabilidades del Estado "la incorporación de las tecnologías de la información y comunicación en el proceso educativo y propiciar el enlace de la enseñanza con las actividades productivas o sociales".

Por su parte, la (Ley Orgánica De Educación Intercultural, 2016), establece algunos principios rectores de la educación basados en fundamentación filosófica, conceptual y constitucional. En ese contexto, en el literal h, del artículo 2 establece el interaprendizaje y multiaprendizaje ambos conceptos se direccionan a fomentar las capacidades humanas a través de la cultura, deporte, acceso a la información, tecnologías, comunicación y conocimiento.

Dentro del Currículo Nacional del Ecuador las clases que imparten los docentes parten de una programación didáctica, aquí las tecnologías de la información y de la comunicación juegan un rol importante porque forman parte del uso habitual como instrumento facilitador para el desarrollo del currículo. Existen algunos objetivos y destrezas orientados al uso responsable de la tecnología en las asignaturas básicas como: matemática, lengua y literatura, ciencias naturales, estudios sociales, Educación Cultural y Artística e incluso en los ámbitos de aprendizaje en inicial y preparatoria.

Por mencionar algunos, en Educación Cultural y Artística se recomienda utilizar medios audiovisuales y tecnologías para el disfrute total del arte y cultura; en ciencias naturales la búsqueda y análisis de las experiencias sobre los fenómenos y hechos naturales, en el área de matemática profundizar temáticas con la investigación en internet con énfasis en la búsqueda de fuentes confiables, entre otros.

Por lo tanto, partiendo de ese análisis el Estado Ecuatoriano en sus leyes establece la aplicación de tecnologías para fines educativos, sin embargo, la realidad es diferente. Muchas instituciones a nivel de País no cuentan con la infraestructura y equipamiento 
tecnológico para garantizar los derechos constitucionales, fomentar la investigación y divulgación científica. Incluso las instituciones educativas que tienen los equipamientos suficientes, aún no ejecutan proyectos o programas orientados hacia el cumplimiento de objetivos establecidos en la ley, sea por el desconocimiento de los docentes para fortalecer dichas competencias digitales en los estudiantes o por la falta de recursos tecnológicos en las escuelas.

Desde ese punto de vista, es necesario abordar los conceptos de competencias digitales. Según (Levano-Francia et al. 2019), son instrumentos que permiten mejorar o transformar actitudes, conocimiento y procesos en las personas para adquirir habilidades que faciliten la transferencia de conocimientos y generen innovación. En el ámbito educativo se espera que los niños durante todo su proceso de formación fortalezcan las competencias digitales necesarias para asumir de manera ética y responsable los problemas que se susciten.

Si bien los conceptos de competencia digital y alfabetización digital se han mencionado con mayor frecuencia en algunos aspectos (Pettersson, 2018), concibe a la competencia digital como el conjunto de habilidades y alfabetizaciones necesarias para que los individuos aprendan y naveguen por el conocimiento digitalizado de la sociedad. Sin embargo, (Spante et al. 2018), indican que, a menudo se emplean como sinónimos, pero tienen orígenes y significados diferentes.

\section{Modelos de Alfabetización Informacional.}

Mencionar sobre Alfabetización Informacional es más complejo que el simple manejo de computadoras en una biblioteca escolar, su aplicación implica una serie de procedimientos. A lo largo de la historia se han establecido una diversidad de modelos para la búsqueda de la información, pero recalcar su importancia en la educación es el primer paso a seguir para que los estudiantes se sientan motivados todo el tiempo.

Según (García-Elías, 2021), el modelo big 6 es una estrategia de investigación que se sustenta en el análisis de la información para solucionar problemas, está constituido por 
seis pasos tales como: 1. Definición del problema o tema de interés, 2. búsqueda de recursos para la búsqueda de información, 3. localización y acceso, 4. análisis de la información, 5. síntesis y organización de la información y 6 . evaluación. Desde ese punto de vista, es correcto aplicar un proceso que organice la estructura mental de los estudiantes, de tal manera que su ejecución sea lo más sencillo posible. Así mismo, es necesario que el docente guié paso a paso durante la enseñanza de alfabetización informacional a los niños para un correcto resultado.

Como recomendaciones generales añadidas al proceso anterior son: al momento de buscar la información en los navegadores correspondientes utilizar palabras clave del problema de esta manera, la búsqueda será más precisa. Además, emplear buscadores boléanos como el "AND" incluyendo todos los términos en la búsqueda, "OR" contener al menos uno de los términos y "NOT" excluir términos, para reducir la información y gestionarla de manera pertinente.

Sin embargo, existen otros modelos para la enseñanza de Alfabetización Informacional. (Huaillani-Chavez, 2020), menciona al modelo de las siete "caras" o categorías de Bruce el cual se constituye por siete categorías de la siguiente manera: 1 . Concepción basada en las Tecnologías de la información, 2. fuentes de información, 3. información como proceso, 4. control de la información, 5. construcción del conocimiento, 6. extensión del conocimiento, 7. Concepción basada en el saber. Por lo tanto, cada una de las etapas comprende una serie de indicaciones para lograr el objetivo.

Este modelo lo que busca es identificar la disponibilidad de las TIC, es decir, cada persona debe ser capaz de reconocer su capacidad para usar la tecnología en contextos comunicativos e informativos. Además, busca conocer la existencia de diversas fuentes de información unas más confiables que otras, y para eso se debe utilizar estrategias que le permitan obtener información verídica acorde a los objetivos de la investigación. Para el control de la información resulta necesario el uso de gestores bibliográficos como Mendeley o Zotero, de esa manera toda la información se convierte en un bagaje de conocimientos fundamentados en la ética y valores. 
En ese sentido, es importante capacitar a los docentes sobre modelos de Alfabetización Informacional de manera que, puedan replicar las estrategias a los estudiantes y sean ellos quienes construyan su conocimiento analizando, leyendo y gestionando la información. Actualmente es preocupante la situación de muchos jóvenes al elaborar una consulta o un proyecto, cómo desconocen del proceso adecuado usan la información sin utilizar la cita correspondiente, o simplemente carecen de conocimiento sobre fuentes de información confiable.

\section{Storytelling}

El Storytelling es considerado una estrategia ideal para fortalecer los aprendizajes. Así pues (Benavides-Bailón \& Mendoza-Lino, 2020), manifiestan que antes de crearse las escuelas, ya los abuelos comentaban historias a los miembros de la familia y comunidad. Quizás de antecedentes sobre la creación del universo, luchas de pueblos, conquistas entre otros. Desde esa mirada, el Storytelling trata de dinamizar la forma de aprender en los niños y niñas destacando el aspecto socioemocional, (Hermann-Acosta, 2020), revela que, una de las características del Storytelling es lo emocional y experiencial, creándose un hilo conductor entre la historia y vivencias personales de los que escuchan.

\section{MÉTODO}

La presente investigación es de tipo descriptiva con un diseño no experimental, posee un enfoque mixto, con cohorte transversal. El estudio se lo realizó en la Escuela de Educación Básica Particular Hispanoamericano ubicada en la ciudad de Santa Rosa, provincia de El Oro, Ecuador la cual tiene una población estudiantil de 194 estudiantes desde el subnivel Inicial I hasta séptimo año EGB.

El estudio se fundamentó en el método estadístico pues se aplicó encuestas a la muestra estratificada de 29 padres de familia correspondientes a quinto, sexto y séptimo año de Educación General Básica, dicho instrumento de recolección de datos estuvo conformado 
por 18 preguntas el cual fue validado a través de un análisis de datos con el Alfa de Cronbach, dando como resultado un coeficiente de 0.752 .

Así mismo, se aplicó instrumentos como el análisis de revisión bibliográfica sobre los fundamentos teóricos de Alfabetización Informacional. Además, entrevistas dirigidas a 3 docentes del subnivel media, 1 docente de la asignatura de computación y a los directivos de la institución para conocer la pertinencia de la investigación en la institución educativa. Para el análisis de los datos se aplicó estadística Descriptiva pues luego de estudiar el comportamiento de las variables se realizó un análisis de normalidad el cual demostró que las 18 variables son paramétricas, es decir H0 (menor a 0.05). Se empleó el Software SPSS para aplicar tablas de frecuencias y análisis de Chi Cuadrado dando respuesta al objetivo de la investigación (Erazo-Álvarez, 2018).

\section{RESULTADOS}

En base al objetivo de investigación sobre "analizar el nivel de conocimiento de Alfabetización Informacional en los estudiantes y docentes de Educación Básica subnivel Media para el desarrollo de competencias digitales", y considerando que, conocer sobre Alfabetización Informacional no basta con ingresar al navegador y buscar la información, se presentan los siguientes resultados:

\section{Tabla 1.}

Análisis Chi cuadrado de Pearson.

\begin{tabular}{|c|c|c|c|c|c|}
\hline & & \multicolumn{4}{|c|}{ Tiempo en actividades con Internet (Investigación y } \\
\hline & & \multicolumn{3}{|c|}{ búsqueda de información) } & \multirow{2}{*}{ Total } \\
\hline & & Poco & Suficiente & Mucho & \\
\hline Lectura, análisis & Nunca & 1 & 0 & 0 & 1 \\
\hline y selección de la & Algunas veces & 4 & 3 & 1 & 8 \\
\hline \multirow[t]{2}{*}{ información } & Frecuentemente & 1 & 6 & 3 & 10 \\
\hline & Siempre & 0 & 5 & 5 & 10 \\
\hline \multirow[t]{2}{*}{ Total } & & 6 & 14 & 9 & 29 \\
\hline & & & $\mathrm{gl}$ & Sig & (bilat \\
\hline
\end{tabular}


Revista Arbitrada Interdisciplinaria KOINONIA

Año VI. Vol VI. N³. Edición Especial: Educación II. 2021

Hecho el depósito de Ley: FA2016000010 ISSN: 2542-3088

FUNDACIÓN KOINONIA (F.K). Santa Ana de Coro. Venezuela.

Karla Esthefanía Jaén-Armijos; Sandra Elizabeth Mena-Clerque

\begin{tabular}{cccc}
\hline Chi-cuadrado de Pearson & $12,308^{\mathrm{a}}$ & 6 & 0,055 \\
Razón de verosimilitudes & 12,948 & 6 & 0,044 \\
Asociación lineal por lineal & 8,664 & 1 & 0,003 \\
N de casos válidos & 29 & & \\
\hline
\end{tabular}

Fuente: Cuestionario.

Según la tabla 1 sobre el análisis de Chi - cuadrado, en relación a la lectura, análisis, selección de la información y el tiempo en actividades con Internet (Investigación y búsqueda de información) se puede observar que el coeficiente de Pearson es de 0.055. Por lo tanto, se concluye que ambas variables tienen relación puesto que, conocer sobre Alfabetización Informacional se vincula directamente con las habilidades del pensamiento, las cuales necesitan de la capacidad lectora de los individuos para poder desarrollar la competencia de observar, buscar, leer, comparar, analizar, clasificar, seleccionar y gestionar la información de manera correcta.

De tal manera que, los estudiantes que pasan más tiempo en internet para actividades de investigación, muy probablemente sigan los procesos adecuados de Alfabetización Informacional en comparación a aquellos estudiantes que dedican su tiempo para el entretenimiento, redes sociales y juegos. Según los resultados, de los 9 estudiantes que pasan mucho tiempo en internet para investigar y buscar información, 5 manifiestan que siempre y frecuentemente leen, analizan y seleccionan la información. Sin embargo, a pesar de que 14 estudiantes pasan tiempo suficiente en internet para la investigación sólo 6 estudiantes frecuentemente leen, analizan y seleccionan la información de manera adecuada, frente a 3 estudiantes que algunas veces lo hacen. Esto representa un factor negativo para la Alfabetización Informacional, pues un correcto procedimiento se fundamenta en leer siempre la información que se encuentra en la Web, incluyendo la búsqueda en varias fuentes de información para así analizarla y posteriormente seleccionarla. 
Tabla 2.

Análisis Chi cuadrado de Pearson.

\begin{tabular}{|c|c|c|c|c|c|c|}
\hline & & \multicolumn{4}{|c|}{ Lectura y análisis de la información } & \multirow{2}{*}{ Total } \\
\hline & & Nunca & Algunas veces & Frecuentemente & Siempre & \\
\hline Citación de & Nunca & 0 & 6 & 2 & 3 & 11 \\
\hline \multirow[t]{3}{*}{ autores } & Algunas veces & 1 & 1 & 4 & 4 & 10 \\
\hline & Frecuentemente & 0 & 0 & 4 & 2 & 6 \\
\hline & Siempre & 0 & 1 & 0 & 1 & 2 \\
\hline \multirow{2}{*}{\multicolumn{2}{|c|}{ Total }} & 1 & 8 & 10 & 10 & 29 \\
\hline & & Valor & \multicolumn{2}{|c|}{ gl } & \multicolumn{2}{|c|}{ Sig. asintótica (bilateral) } \\
\hline \multicolumn{2}{|c|}{ Chi-cuadrado de Pearson } & $11,763^{\mathrm{a}}$ & \multicolumn{2}{|c|}{9} & \multicolumn{2}{|c|}{0,227} \\
\hline \multicolumn{2}{|c|}{ Razón de verosimilitudes } & 13,759 & \multicolumn{2}{|r|}{9} & \multicolumn{2}{|l|}{0,131} \\
\hline \multicolumn{2}{|c|}{ Asociación lineal por lineal } & 1,274 & \multicolumn{2}{|c|}{1} & \multicolumn{2}{|l|}{0,259} \\
\hline \multicolumn{2}{|c|}{$\mathrm{N}$ de casos válidos } & 29 & & & & \\
\hline
\end{tabular}

Fuente: Cuestionario.

Según la tabla 2 sobre el análisis de Chi - cuadrado, en relación a la lectura, análisis, selección de la información y la citación de autores se puede observar que el coeficiente de Pearson es de 0.227. Por lo tanto, las variables no tienen relación entre ellas, porque el hecho de leer, analizar y seleccionar la información no significa que todos los niños sigan el proceso correcto de citar correctamente.

Desde ese punto de vista, los resultados reflejan que, de los 8 estudiantes que algunas veces leen, 6 nunca citan al autor. De los 10 estudiantes que frecuentemente leen, 4 algunas veces y frecuentemente citan al autor. $Y$ de los 10 estudiantes que siempre leen, 4 citan algunas veces y 3 estudiantes nunca citan. Esto demuestra que, independientemente de su conocimiento sobre Alfabetización Informacional no se está siguiendo un proceso correcto y ético de la información, dando como consecuencia que los estudiantes copien y peguen, apropiandose de información que no les pertenece.

Por otro lado es importante que los estudiantes al momento de documentar la información sigan buenas prácticas para evitar el plagio de información. Es por ello que, los gestores 
bibliográficos son considerados una herramienta esencial para citar el origen de los datos (Fernandez-Izquierdo, 2018).

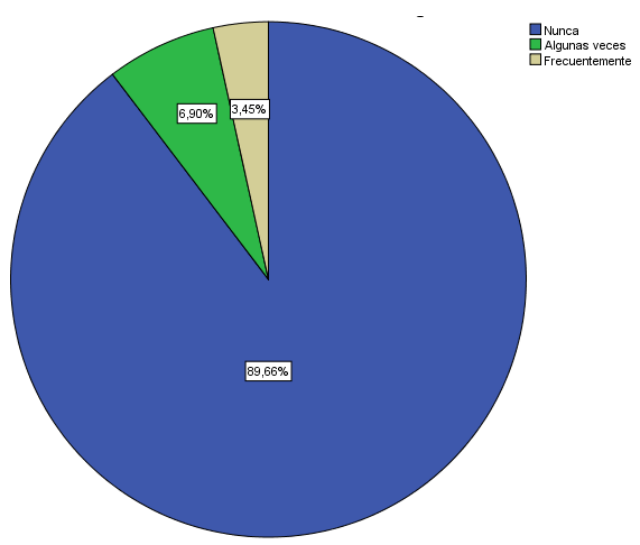

Figura 1. Uso de gestores bibliográficos Fuente: Cuestionario.

Como se puede observar en la Figura 1 el 89,66\% de la población estudiantil nunca utiliza gestores bibliográficos como Zotero o Mendeley, frente a un $6.90 \%$ algunas veces, y $3.45 \%$ frecuentemente. En definitiva, la mayoría de estudiantes no estan empleando gestores bibliográficos como recurso esencial de todo proceso de Alfabetización Informacional. Para ello es necesario recalcar que, los gestores bibliográficos ayudan a organizar referencias, permiten almacenar gráficos, textos completos, y citar de la forma más eficiente posible.

La entrevista fue aplicada a docentes de quinto, sexto y séptimo año Educación General Básica y área de computación. Por lo tanto, en base a los criterios emitidos en la Unidad de análisis, categorías del conocimiento de alfabetización y competencias digitales, se observan los siguientes resultados. 
Revista Arbitrada Interdisciplinaria KOINONIA

Año VI. Vol VI. N³. Edición Especial: Educación II. 2021

Hecho el depósito de Ley: FA2016000010 ISSN: 2542-3088

FUNDACIÓN KOINONIA (F.K). Santa Ana de Coro. Venezuela.

Karla Esthefanía Jaén-Armijos; Sandra Elizabeth Mena-Clerque

\section{Tabla 3.}

Resultados de la entrevista a docentes.

\begin{tabular}{|c|c|c|}
\hline Unidad de análisis & Categoría & Segmento \\
\hline \multirow{3}{*}{$\begin{array}{l}\text { Alfabetización } \\
\text { Informacional }\end{array}$} & Nivel de conocimiento & $\begin{array}{l}\text { - No mucho, quizás con otras palabras. } \\
\text { - No, no he escuchado de ese término, solamente } \\
\text { Alfabetización, pero Informacional no. } \\
\text { - Primera vez que escucho ese término. }\end{array}$ \\
\hline & $\begin{array}{l}\text { Importancia Alfabetización } \\
\text { Informacional estudiantes }\end{array}$ & $\begin{array}{l}\text { - Sí, los niños deberían conocer sobre Alfabetización } \\
\text { Informacional. } \\
\text { - Sí, sería conveniente y muy importante } \\
\text { - Sí, sería muy bueno para que a su temprana edad ya } \\
\text { sepan y en un futuro puedan aportar en algo. }\end{array}$ \\
\hline & Capacitación docente & $\begin{array}{l}\text { - Sí, me gustaría recibir la capacitación. } \\
\text { - Sí, sería muy bueno que los docentes conozcan sobre } \\
\text { Alfabetización Informacional. }\end{array}$ \\
\hline \multirow{4}{*}{$\begin{array}{c}\text { Competencia digital en } \\
\text { uso de los datos }\end{array}$} & Fuentes información & $\begin{array}{l}\text { - Google y YouTube. } \\
\text { - En páginas de internet } \\
\text { - Si se trata de artículos mejor en el Google Educativo. } \\
\text { Y Videos en YouTube. }\end{array}$ \\
\hline & Frecuencia búsqueda & $\begin{array}{l}\text { - Siempre le ha gustado investigar y ahora más por la } \\
\text { enseñanza de computación. } \\
\text { - Frecuentemente, de acuerdo cuando se presente la } \\
\text { investigación. } \\
\text { - Casi siempre. } \\
\text { - Siempre, por las clases virtuales. }\end{array}$ \\
\hline & $\begin{array}{l}\text { Proceso búsqueda } \\
\text { información }\end{array}$ & $\begin{array}{l}\text { - De acuerdo al tema, si es concepto de algo me gusta } \\
\text { buscar en varias fuentes. } \\
\text { - Consultar varias fuentes para poder obtener un } \\
\text { objetivo o definición. } \\
\text { - Ingresar en el Google educativo, y buscar el tema que } \\
\text { uno quiere investigar. }\end{array}$ \\
\hline & $\begin{array}{l}\text { Búsqueda información } \\
\text { Estudiantes }\end{array}$ & $\begin{array}{l}\text { - En 7mo EGB les ha enviado a consultar en internet. } \\
\text { - Cuando es conveniente y amerita el caso sí. } \\
\text { - Si, en Lengua y Literatura y Ciencias Naturales. }\end{array}$ \\
\hline
\end{tabular}


- Hasta el momento no, porque recién empieza las clases. Pero años anteriores sí.

\begin{tabular}{cc}
\hline Sugerencia fuentes & - No, en ese sentido la verdad se me ha pasado por alto. \\
información estudiantes & - No, realmente no. Porque quizás todos los niños no \\
& tienen las mismas tecnologías y dispositivos. \\
- Que investiguen en el Google Educativo, pues en el & Google normal la información suele no ser confiable. \\
& - Se les entrega ciertas herramientas para que los niños \\
& puedan buscar pues por lo general seleccionan lo \\
& primero que encuentran. \\
\hline investigaciones estudiantes & - Por lo general copian y pegan la información que \\
& encuentran en internet. \\
& Si la consulta es leer y determinar una definición propia \\
suelen copiar y pegar de varias fuentes. \\
- Al inicio si copiaban y pegaban la información. Luego \\
leyeron y parafrasearon la información, copiando el link \\
de acceso o Webgrafía.
\end{tabular}

\section{Fuente: Entrevistas.}

Analizando los resultados de las entrevistas se puede mencionar que, si bien los docentes han escuchado sobre Alfabetización, el término "Alfabetización Informacional" fue completamente nuevo ellos. Eso demuestra que los docentes como entes promotores de saberes y conocimientos, en cuanto al proceso de analizar, buscar, comparar, y evaluar la información no se está abordando en las aulas. Lo que representa un factor negativo para la Alfabetización Informacional, pues el docente es la pieza fundamental en todo el proceso de enseñanza aprendizaje.

Así mismo, en la pregunta sobre las fuentes de información donde suelen investigar los docentes, estos respondieron que frecuentemente recurrían a Google, YouTube, páginas de internet y solamente una docente recurría a Google Educativo o Google Académico. Además, manifestaron que de acuerdo a la necesidad solían investigar, por ejemplo, en la actualidad por la Emergencia Sanitaria, las clases se desarrollan de forma virtual, lo 
cual exige una formación y capacitación constante del docente para afrontar las demandas educativas.

En cuanto al proceso de búsqueda de información, los docentes textualmente manifestaron que:

- De acuerdo al tema, si es concepto de algo me gusta buscar en varias fuentes.

- Consultar varias fuentes para poder obtener un objetivo o definición.

- Ingresar en el Google educativo, y buscar el tema que uno quiere investigar.

Desde ese punto de vista, los maestros necesitan conocer de un proceso adecuado para buscar y gestionar la información. Porque no se trata solamente de buscar en varias fuentes de información, sino de identificar cuáles fuentes son las más adecuadas y usar técnicas booleanas.

Ahora bien, en relación a las investigaciones realizadas por los estudiantes, y si los docentes recomiendan fuentes de información, la mayoría manifestó que sí han enviado hacer consultas en internet dependiendo de la temática y destreza de estudio. Así mismo, la mayoría de docentes indica que no suelen sugerir fuentes de información a sus estudiantes, ellos lo hacen de manera independiente.

Entre las dificultades más preponderantes al momento de evaluar las investigaciones o consultas son el plagio de información, los docentes manifiestan que generalmente los estudiantes leen la información encontrada, sin embargo, copian y pegan definiciones, extractos y conceptos. Los estudiantes no poseen la capacidad para parafrasear la información, a pesar de siempre recordarles colocar la bibliografía. 
Revista Arbitrada Interdisciplinaria KOINONIA

Año VI. Vol VI. N³. Edición Especial: Educación II. 2021

Hecho el depósito de Ley: FA2016000010 ISSN: 2542-3088

FUNDACIÓN KOINONIA (F.K). Santa Ana de Coro. Venezuela.

Karla Esthefanía Jaén-Armijos; Sandra Elizabeth Mena-Clerque

\section{Tabla 4.}

Resultados de la entrevista al Representante Legal.

\begin{tabular}{|c|c|c|}
\hline Unidad de análisis & Categoría & Segmento \\
\hline \multirow{6}{*}{$\begin{array}{l}\text { Alfabetización } \\
\text { Informacional }\end{array}$} & Nivel de conocimiento & - No he escuchado sobre Alfabetización Informacional \\
\hline & $\begin{array}{l}\text { Importancia Alfabetización } \\
\text { Informacional }\end{array}$ & $\begin{array}{l}\text { - Considero que es importante que los niños sepan } \\
\text { cómo buscar información adecuadamente para que } \\
\text { puedan hacer sus trabajos. }\end{array}$ \\
\hline & $\begin{array}{l}\text { Programa de capacitación } \\
\text { Docentes - estudiantes }\end{array}$ & $\begin{array}{l}\text { - Considero que todo el personal docente de las } \\
\text { instituciones educativas sea particulares o públicos } \\
\text { deberían conocer sobre Alfabetización Informacional. } \\
\text { En nuestra institución considero que falta mejorar en } \\
\text { ese aspecto y vendría bien una capacitación a los } \\
\text { docentes y ellos a su vez enseñen a sus estudiantes. } \\
\text { Sería una excelente propuesta. }\end{array}$ \\
\hline & Aprovechamiento & $\begin{array}{l}\text { - La maestra de computación directamente sí, o por lo } \\
\text { menos conoce un poco más de la materia. Pero los } \\
\text { docentes de áreas básicas no promueven en su } \\
\text { totalidad la Alfabetización Informacional, en cierta } \\
\text { parte con consultas que envían a sus estudiantes. }\end{array}$ \\
\hline & Políticas educativas & $\begin{array}{l}\text { - He leído un poco sobre las leyes que propone el } \\
\text { Ministerio de Educación. Pero específicamente de } \\
\text { Alfabetización digital no. Lo que sí, dentro de la Ley } \\
\text { establece que la asignatura de computación no forma } \\
\text { parte del currículo. }\end{array}$ \\
\hline & $\begin{array}{l}\text { Alfabetización Informacional } \\
\text { Estudiantes }\end{array}$ & $\begin{array}{l}\text { - Un poco se podría decir por lo que se imparte } \\
\text { computación. }\end{array}$ \\
\hline \multirow{2}{*}{$\begin{array}{c}\text { Infraestructura } \\
\text { Educativa }\end{array}$} & $\begin{array}{c}\text { Biblioteca escolar y sala de } \\
\text { computación }\end{array}$ & $\begin{array}{l}\text { - Si, cuenta con sala de computación y se suele utilizar } \\
\text { para biblioteca escolar realizar investigaciones etc. }\end{array}$ \\
\hline & Asignatura computación & $\begin{array}{l}\text { - Si se imparte la asignatura desde 1ero EGB hasta 7mo } \\
\text { EGB, } 2 \text { horas semanales cada grado. }\end{array}$ \\
\hline
\end{tabular}

\section{Fuente: Entrevistas.}


Según los resultados de la entrevista dirigida al directivo de la institución en relación a la infraestructura educativa se puede evidenciar que, la escuela si cuenta con sala de computación, la cual es utilizada como biblioteca escolar al mismo tiempo y también se imparte la asignatura de computación desde primero a séptimo EGB. Esto representa un factor positivo para la Alfabetización Informacional pues, fortalecer las capacidades informacionales en los estudiantes depende de los recursos tecnológicos, dispositivos electrónicos e internet. Sumado a eso, enseñar a los estudiantes computación es primordial para alfabetizar informacionalmente a los estudiantes.

Sin embargo, el representante legal de la institución no conoce sobre Alfabetización Informacional y comparando la entrevista a docentes, donde tampoco han escuchado de ese término, se nota una problemática potencial, porque son los directivos y docentes de la institución quienes promueven dichas competencias informacionales en los estudiantes. Además, tanto docentes como directivos consideran necesario que los niños conozcan el proceso de buscar, identificar, seleccionar, evaluar y gestionar la información adecuadamente para desarrollar sus competencias digitales. Por lo tanto, diseñar un programa de capacitación sobre alfabetización informacional es una excelente propuesta en la investigación.

Definitivamente, la Alfabetización Informacional es más complejo que una simple búsqueda de información en internet. Incluso hablar solamente de "Alfabetización" es aún desconocido, pues su epistemología va más allá que leer y escribir. Para (Manghi-Haquin et al. 2016), alfabetizar no abarca solamente la capacidad de leer y escribir sino implica tener capacidad para comprender y emplear diferentes tipos de información sean estos contextos sociales, personales, familiares y culturales. Desde ese punto de vista, los docentes aun manifestando que sólo conocen el término alfabetizar están mal direccionados, pues la "alfabetización" abarca también gestión de la información. Según (Escobar-Blanco et al. 2016), la Alfabetización Informacional es sinónimo de competencia informacional, la cual define como la capacidad para reconocer el motivo de buscar información, organizarla y utilizarla en la resolución de problemas a lo largo de la 
vida. Acorde con el autor, y en base al estudio realizado en la investigación, se puede sintetizar todo el proceso de Alfabetización Informacional en competencias informacionales.

No obstante, se suele confundir la Alfabetización Informacional con Alfabetización digital, esta última se diferencia por las 5 dimensiones: 1. Conocimiento instrumental de Hardware y software; 2. Conocimiento informacional; 3. Comunicación con las TIC; 4. Conciencia del uso de las tecnologías; 5. Construcción de la identidad digital. (AvilaMuñoz, 2016).

\section{PROPUESTA}

Con miras a desarrollar las competencias digitales sobre el uso y gestión de la información en los estudiantes y docentes de la institución. Y luego de un análisis riguroso de los resultados de la investigación donde se pudo evidenciar un desconocimiento de procesos de Alfabetización Informacional, se establece la siguiente propuesta de solución la cual posee un enfoque innovador con principios formativos, flexibles y adaptables al contexto educativo. Cabe mencionar que, la propuesta está dirigida en primera instancia a los docentes de la institución para que a su vez puedan enseñar a sus estudiantes.

Objetivo General: Diseñar un programa de formación en Alfabetización Informacional basado en el Modelo KALFIN 4 para desarrollar competencias digitales e informacionales en los docentes.

Estimación del tiempo: 2 meses, 3 veces por semana (Total 24 sesiones de trabajo) Medio: Sincrónica y asincrónica

\section{Perfil del Programa de formación}

Paso 1. Aplicar un Pre - test sobre Alfabetización Informacional para evaluar el nivel de competencias digitales e informacionales de los docentes. Para el diseño de los cuestionarios de evaluación diagnóstica se utilizará la plataforma Kahoot como 
instrumento didáctico y se explicará a los docentes la forma de participación. Además, la introducción al programa tendrá las siguientes preguntas:

- ¿Cómo buscas la información?

- ¿Qué estrategias utilizas para buscar información confiable?

- ¿Por qué existe la necesidad de buscar información?

- ¿Cómo gestionas y evalúas la información?

Paso 2. Explicar el programa de Alfabetización Informacional a los docentes a través de un "Storytelling problematizador" elaborado en la plataforma Genially. Donde se contemple los siguientes aspectos:

- Objetivos

- Contenidos: Definición de Alfabetización Informacional, competencias informacionales, importancia, integración en el Currículo, entre otros.

- Metodología de estudio: Aprendizaje colaborativo, Aprendizaje basado en proyectos, comprensión lectora, entre otros.

- Evaluación

Paso 3. Iniciar los talleres utilizando estrategias activas enfocadas en el modelo KALFIN 4. Cuyas etapas contemplan lo siguiente:

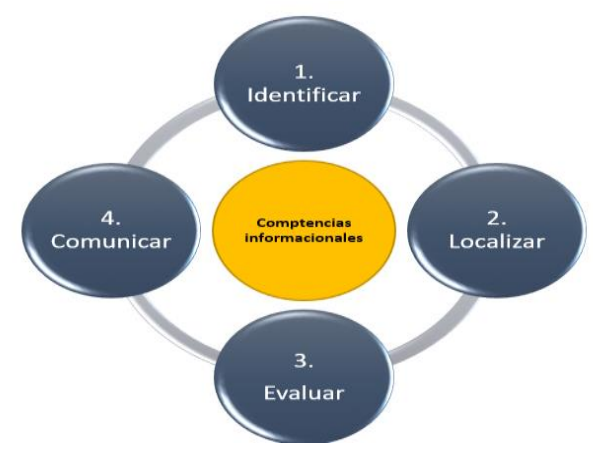

Figura 2. Modelo KALFIN 4. Elaboración: Los autores. 
El modelo KALFIN 4 es una recopilación de fundamentos epistemológicos y metodológicos sobre competencias informacionales el cual se direcciona a un proceso pertinente para resolver problemas de investigación, lograr objetivos de aprendizaje y desarrollar habilidades informacionales para la vida diaria. Está constituido por 4 competencias fundamentales: identificar, localizar, evaluar y comunicar. Así mismo, el modelo KALFIN 4 puede aplicarse en diferentes grados de profundización dependiendo del grupo a quién va dirigido, si el caso es de estudiantes se utilizarán herramientas didácticas para aplicar las 4 competencias.

Competencia 1: Identificar. - Esta primera etapa consiste en identificar el problema y tema de investigación, que responda a la necesidad de buscar la información. Desde ese punto de vista, los docentes deberán identificar un problema educativo por mencionar algunos: El rol de la familia en la escuela, repercusión de la inasistencia a las clases virtuales, la COVID 19 y su incidencia en la educación, entre otros.

Competencia 2: Localizar. - La segunda etapa consiste en 2 pasos. El primer paso "acceder a las diferentes fuentes de información", aquí los docentes contarán con diferentes fuentes como: Google Académico, repositorios de Universidades, Google, Páginas Web, bases de datos, libros entre otros. Cabe señalar la necesidad de utilizar palabras clave y filtros de información para que la búsqueda sea más precisa.

El segundo paso "analizar y seleccionar la información", aquí los docentes deberán emplear habilidades lectoras para posteriormente seleccionar la información más relevante que estén acordes a su objetivo. Como sugerencia podrán utilizar buscadores boléanos como el "AND" incluyendo todos los términos en la búsqueda, "OR" contener al menos uno de los términos y "NOT" excluir términos, para reducir la información y extraer la más esencial.

Competencia 3: Evaluar. - La tercera etapa es la más importante, pues se trata de juzgar y valorar la información seleccionada. Aquí se establecen criterios o estrategias para verificar la fiabilidad de la información tales como: contenido, autores, fecha de publicación, fuentes adicionales, información de contacto entre otros. Desde esa mirada, 
desarrollar esta competencia es imprescindible porque solo así, se logrará obtener información veraz y contrastada. Por otra parte, analizar la calidad y pertinencia de la información depende del docente investigador pues él decide si la información es coherente al objetivo de estudio.

Competencia 4: Comunicar. - La cuarta etapa se enfoca en difundir la información de forma ética y responsable. Para ello es necesario tener en cuenta que toda la información encontrada en internet no es de absoluta autoría del investigador, por lo tanto, utilizar los gestores bibliográficos será una herramienta eficaz para poder citar la información y comunicarla de forma ética hacia la sociedad.

Paso 4. Finalmente, luego de la ejecución de talleres con los docentes, se aplicará un Pos - test para evaluar el alcance de la formación sobre Alfabetización Informacional y analizar si nivel de competencias digitales e informacionales de los docentes mejoró en relación al Pre - test.

Es fundamental que, los directivos se involucren permanentemente durante todo el proceso de formación en Alfabetización Informacional, y se establezcan políticas internas en la institución sobre el fortalecimiento de habilidades y competencias digitales en cuanto al uso y gestión de la información. Solo así, la presente propuesta será trascendental en la mejora de la calidad de la educación.

\section{CONCLUSIONES}

En definitiva, la Alfabetización Informacional constituye uno de los factores claves para mejorar la calidad de la educación, puesto que innovar en procesos que faciliten el acceso, almacenamiento, aplicación y transmisión de la información permitirá que muchos niños, niñas y adolescentes del sistema educativo alcancen los niveles de pensamiento superior y cumplan los objetivos de aprendizaje.

Según el estudio realizado, existe un deficiente proceso para indagar, examinar y evaluar la información, por lo tanto, se puede concluir que, a pesar de que la mayoría de 
estudiantes dedica su tiempo en internet para investigar, no lo están haciendo de forma correcta. Además, tanto estudiantes, docentes y directivos desconocen el término "Alfabetización Informacional" así como las competencias digitales en el uso y gestión de la información.

Por lo tanto, aplicar el modelo KALFIN 4 como propuesta innovadora para desarrollar las competencias informacionales permitirá que tanto docentes como estudiantes sepan cuándo, y cómo acceder a la información. Es preciso mencionar que, al ser un modelo adaptable a cualquier contexto educativo se puede aplicar en distintos niveles de profundización. Finalmente, el rol de los directivos resulta imprescindible para motivar a los docentes en el cambio de paradigmas informacionales.

\section{FINANCIAMIENTO}

No monetario.

\section{AGRADECIMIENTO}

A la Jefatura de Posgrados de la Universidad Católica de Cuenca por permitir el desarrollo y fomento de la investigación.

\section{REFERENCIAS CONSULTADAS}

Avila-Muñoz, P. (2016). Construcción de ciudadanía digital : un reto para la Educación [Construction of digital citizenship: a challenge for Education]. Suplemento SIGNOS $E A D$. https://p3.usal.edu.ar/index.php/supsignosead/article/view/3666

Benavides-Bailón, J. M. ., \& Mendoza-Lino, P. (2020). El Storytelling en la Educación Superior: un Análisis del Impacto y Pertinencia de la Narración de Historias en el Proceso Formativo [Storytelling in Higher Education: An Analysis of the Impact and Relevance of Storytelling in the Formative Process]. Revista Científica Hallazgos21, 5(2), 149-161.

Constitución de la Republica del Ecuador. (2008). Registro Oficial 449 de 20-oct-2008 Ultima modificación: 13-jul-2011. https://n9.cl/hd0q 
Revista Arbitrada Interdisciplinaria KOINONIA

Año VI. Vol VI. N³. Edición Especial: Educación II. 2021

Hecho el depósito de Ley: FA2016000010

ISSN: 2542-3088

FUNDACIÓN KOINONIA (F.K). Santa Ana de Coro. Venezuela.

Karla Esthefanía Jaén-Armijos; Sandra Elizabeth Mena-Clerque

Chetty, K., Aneja, U., Mishra, V., Gcora, N. \& Josie, J. (2018). Bridging the digital divide in the G20: skills for the new age. Economics, 12(1), 20180024. https://doi.org/10.5018/economics-ejournal.ja.2018-24

Erazo-Álvarez, J. C. (2018). El capital intelectual en el clúster de cuero y calzado de la Provincia de Tungurahua [Intellectual capital in the leather and footwear cluster of the Province of Tungurahua]. Killkana Social, 2(3), 109-114. https://doi.org/10.26871/killkana social.v2i3.333

Escobar-Blanco, L. M., Lauzurica González, A., Soler Cárdenas, S. F., Secada Cárdenas, E., González Herrera, O., \& Tápanes Galvan, W. (2016). Las Competencias Informacionales en los profesores de la Universidad de Ciencias Médicas de Matanzas [Informational competences of professors of the Medical Sciences University of Matanzas]. Revista Médica Electrónica, 38(4), 543-552

Fernandez-Aragones, A., \& Camon, L. (2020). Educando contra la posverdad. La alfabetización informacional como estrategia para hacer resilentes a los menores en el consumo digital [Educating against post-truth. Information literacy as a strategy to make minors resilient in digital consumption]. ZER: Revista De Estudios De Comunicación $=$ Komunikazio Ikasketen Aldizkaria, 25(48). https://doi.org/10.1387/zer.21576

Fernandez-Izquierdo, F. (2018). Una aproximación a los instrumentos metodológicos digitales: los gestores bibliográficos [An approach to digital methodological instruments: bibliographic managers]. En Pons, A. \& Eiroa, M. eds. Historia digital: una apuesta del siglo XXI [Digital history: a bet 21st century]. Ayer, 2018(110), 5182. https://n9.cl/vdzy3

García-Ávila, S. (2017). Alfabetización Digital [Digital Literacy]. Razón y Palabra, 21(98),66-81.

García-Elías, A. (2021). Unidad curricular: la integración de las competencias de información al currículo utilizando estrategias innovadoras en la biblioteca escolar [Curriculum unit: the integration of information competencies into the curriculum using innovative strategies in the school library]. Acceso. Revista Puertorriqueña De Bibliotecología Y Documentación, 2(1), 18

González-López, M., Machin Mastromatteo, J. D., \& Tarango, J. (2019). Alfabetización Informacional: enseñanza y desarrollo de su competencia en la educación básica [Information Literacy: teaching and developing their competence in basic education]. E-Ciencias de La Información, 9. https://doi.org/10.15517/eci.v9i2.35774 
Revista Arbitrada Interdisciplinaria KOINONIA

Año VI. Vol VI. N³. Edición Especial: Educación II. 2021

Hecho el depósito de Ley: FA2016000010

ISSN: 2542-3088

FUNDACIÓN KOINONIA (F.K). Santa Ana de Coro. Venezuela.

Karla Esthefanía Jaén-Armijos; Sandra Elizabeth Mena-Clerque

Gutiérrez, K., García, V., \& Aquino, S. (2016). El desarrollo de las competencias digitales de niños de quinto y sexto año en el marco del programa de MiCompu. Mx en Tabasco [The development of the digital skills of fifth and sixth year children within the framework of the MiCompu program. Mx in Tabasc]. Perspectivas Docentes, 61.

Hermann-Acosta, A. (2020). Storytelling y comunicación multidireccional: una estrategia formativa para la era digital [Storytelling and multidirectional communication: a training strategy for the digital age]. URU. Revista de Comunicación y Cultura, 3043. https://doi.org/10.32719/6312514.2020.3.3

Huaillani-Chavez, S. (2020). Influencia de un programa de alfabetización informacional para el desarrollo de habilidades informativas en los profesionales de un instituto pediátrico [Influence of an information literacy program for the development of information skills in the profess]. Revista Cubana de Información En Ciencias de La Salud, 31(2), 1-16.

Levano-Francia, L., Sanchez Diaz, S., Guillen Aparicio, P., Tello Cabello, S., Herrera Paico, N., \& Collantes Inga, Z. (2019). Competencias digitales y educación [Digital skills and education]. Propósitos y Representaciones, 7(2), 569-588. http://dx.doi.org/10.20511/pyr2019.v7n2.329

Ley Orgánica De Educación Intercultural. (2016). Función Ejecutiva Presidencia De La República Ley Orgánica De Educación Intercultural [Executive Function Presidency of the Republic Organic Law of Intercultural Education]. https://n9.cl/1fo3

Lucas, D., y Calle, R. (2019). La alfabetización informacional en la innovación curricular: estrategias pedagógicas a partir del empleo de la biblioteca escolar [Information literacy in curricular innovation: pedagogical strategies based on the use of the school library]. Rehuso, 4(2), 69-81.

Manghi-Haquin, D, Crespo-Allende, N, Bustos Ibarra, A, \& Haas Prieto, V. (2016). Concepto de alfabetización: ejes de tensión y formación de profesores [The Concept of Literacy: Sources of Tension and Teacher Training]. Revista electrónica de investigación educativa, 18(2), 79-91.

Morales-Camargo, C. L. (2020). Diseño, implementación y evaluación de un plan de alfabetización informacional para la vinculación de TIC al aula de clase en el área de lengua castellana de la I.E.C.S. [Design, implementation and evaluation of an information literacy plan to link ICT to the classroom in the Spanish language area of the I.E.C.S.]. http://hdl.handle.net/11349/25534 
Revista Arbitrada Interdisciplinaria KOINONIA

Año VI. Vol VI. N³. Edición Especial: Educación II. 2021

Hecho el depósito de Ley: FA2016000010 ISSN: 2542-3088

FUNDACIÓN KOINONIA (F.K). Santa Ana de Coro. Venezuela.

Karla Esthefanía Jaén-Armijos; Sandra Elizabeth Mena-Clerque

Pettersson, F. (2018). On the issues of digital competence in educational contexts - a review of literature. Education and Information Technologies, 23(3), 1005-1021. https://doi.org/10.1007/s10639-017-9649-3

Recio-Pineda, S. R. (2019). Velocidade de leitura, prosódia e resultados de compreensão. Revista Horizontes De Linguistica Aplicada, 18(2), 115-137. https://doi.org/10.26512/rhla.v18i2.26979

Spante, M., Hashemi, S. S., Lundin, M., \& Algers, A. (2018). Digital competence and digital literacy in higher education research: Systematic review of concept use. Cogent Education, 5(1), 1-21. https://doi.org/10.1080/2331186X.2018.1519143

(C2021 por los autores. Este artículo es de acceso abierto y distribuido según los términos y condiciones de la licencia Creative Commons Atribución-NoComercial-Compartirlgual 4.0 Internacional (CC BY-NC-SA 4.0)

(https://creativecommons.org/licenses/by-nc-sa/4.0/). 